\title{
REGISTRO DE SEÑALES FÍSICAS UTILIZANDO PC
}

\author{
Sara Sáenz C. , José González E ${ }^{* *}$ y Eusebio García **
}

\begin{abstract}
It described steps to acquire physical signals using the ADC0808 chip. The use of hardware that becomes obsolescence do to development hardware and software is put foreword.
\end{abstract}

\begin{abstract}
RESUMEN
La intención de este artículo es esbozar el proceso seguido para efectuar registros de señales físicas en la computadora utilizando el conversor ADCO8O8. Igualmente se propone rescatar y aprovechar los equipos de cómputo que se encuentran almacenados y en desuso en diversas instituciones por la pronta obsolescencia del "hardware" y del "software" de los mismos.
\end{abstract}

\section{INTRODUCCIÓN}

Es evidente la importancia que ha cobrado la computadora tanto en el ámbito de la industria y el comercio como en el ámbito educativo. La computadora ha impregnado la tecnología jugando un papel definitivo frente al progreso, la comunicación e incluso el conocimiento. Sus aplicaciones abarcan potencialmente a todas las actividades humanas: recreación, educación, producción, etc.

Dentro del terreno educativo; se ha convertido en un medio de instrucción en diferentes áreas y niveles ${ }^{1}$. Ya sea, cuando se trate de memorizar algún conocimiento mediante el entrenamiento y práctica ó en lo concerniente a un auto-aprendizaje recurriendo a los sistemas de agentes ${ }^{2}$. En este último aspecto hay grandes esfuerzos para dotar a las máquinas de inteligencia con el fin de que desarrollen tareas como si se tratara de un humano ${ }^{3}$.

Pero además de estas aplicaciones; en el ámbito educativo hay otro campo en el que la computadora juega un papel relevante y consiste en usarlo como instrumento de registro y control simultáneo de fenómenos físicos tales como temperatura, velocidad, posición, etc.,

\footnotetext{
*Estudiante Maestría en Docencia de la Física. Departamento de Física, Facultad de Ciencia y Tecnología. Universidad Pedagógica Nacional.

** Profesores Facultad de Ciencia y Tecnología. Departamento de Física. Universidad Pedagógica Nacional. Santa Fe de Bogotá, D.C.

${ }^{1}$ Documento para la acreditación previa del programa de licenciatura en física. Departamento de Física. Universidad Pedagógica Nacional. Santa Fe de Bogotá, 1999.

${ }^{2}$ Russel 5., Norvig P. 1996. Inteligencia Artificial (un en foque moderno). Prentice Hall Hispanoamericana S.A. México.
}

${ }^{3}$ Papert, 5. 1981. Desafio a lamente. Ediciones Galápago. Buenos Aires. 
lo cual, resulta muy práctico y asequible a todas las personas coadyuvando al aprendizaje y ofreciendo la posibilidad de registrar las señales analógicas de determinado fenómeno físico.

Realizar cualquier registro es una tarea que requiere de un proceso en el que se conoce más a fondo la computadora como herramienta para acercarse a los hechos físicos. Por esto resulta interesante analizar el proceso mediante el cual es posible efectuar el registro de determinada señal física.

\section{INTERFASE}

En términos generales cualquier variable física puede llevarse al interior de la computadora y procesarse con la misma facilidad y eficiencia con la cual se elabora una contabilidad en una empresa o un registro de calificaciones en una institución escolar ${ }^{4}$.

Pero como casi el cien por cien de los fenómenos físicos son de naturaleza análoga o de variación continua en el tiempo, y la computadora sólo maneja variables de tipo digital que toman dos valores definidos: Voltaje alto (1) y voltaje bajo (O); se necesita realizar una interface entre el mundo físico y la computadora que consiste en:

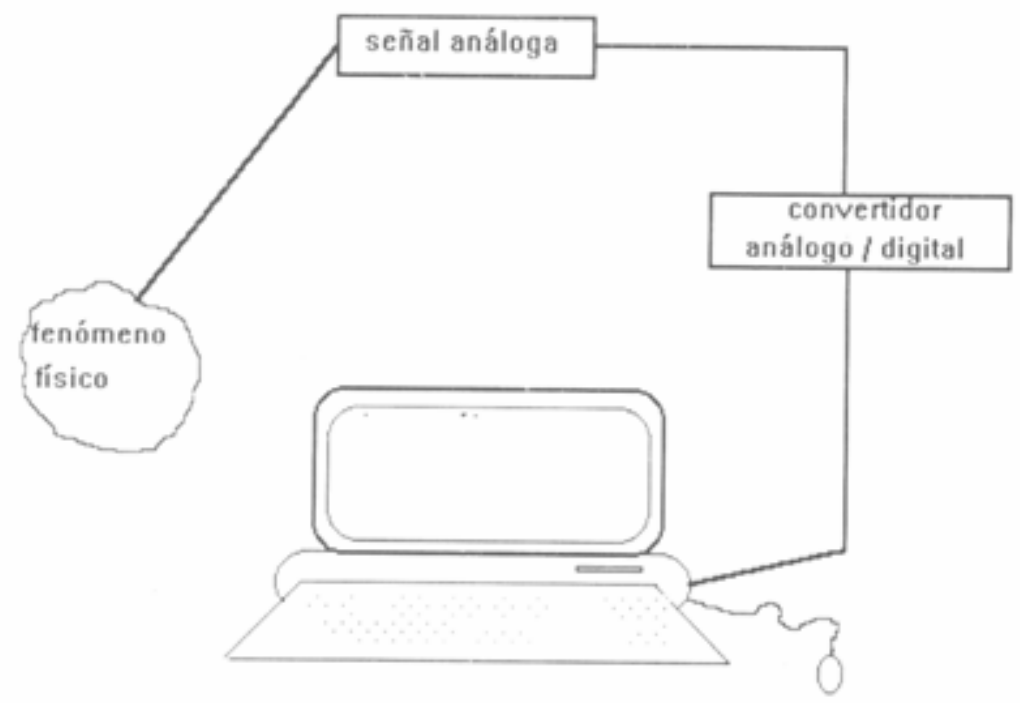

Figura 1. Diagrama que representa la comunicación entre el fenómeno y la computadora ${ }^{5}$

- Dado el fenómeno físico usar el transductor adecuado que convierta la variable física, que se desea medir, en una señal eléctrica.

- Si la señal es muy débil se usa un amplificador que además de incrementar en magnitud la señal obtenida del transductor; puede filtrarla, procesarla y en algunos casos corregirla.

- Una vez lista la señal debe ser convertida en digital, que es el tipo de señal que entiende la computadora. Los circuitos que desarrollan esta función se llaman

\footnotetext{
${ }^{4}$ CEKIT. 1994. Electrónica \& Computadores. Año 1, N ${ }^{0} 1$. Pereira, Colombia.

${ }^{5}$ GONZÁLEZ, O., Yate, R. 1997. Adquisición de señales análogas por computador. Monografía de Grado. Departamento de Física. Universidad Pedagógica Nacional.
} 
Convertidores Análogo-Digitales; de los cuales existen diferentes tipos con sus respectivas características (ver figura 1). Una buena opción es el convertidor ADC0808 que consta de 28 pines de los cuales 8 corresponden a los canales que posibilitan la captura de las señales, aunque sólo puede leer una a la vez, y dispone además de un selector de 3 líneas digitales que mediante el código binario permite escoger el canal que se va a leer. Este convertidor ofrece alta rapidez, alta precisión, mínima dependencia de temperatura y bajo consumo de potencia. Todas estas características resultan ideales para aplicaciones de control y captura de señales ${ }^{6}$.

- Después de la conversión se tiene una palabra de 8 bits que debe entrar a la computadora; ya sea por:

a. El puerto serial cuyo papel es recibir la palabra de 8 bits dada por el convertidor en una forma serial, es decir, bit por bit (se requiere transformar una presentación de una palabra paralela a una forma serial).

b. El bus interno.

o. El puerto paralelo (o de impresora) resulta más sencillo de usar cuando se inician las prácticas con sistemas de control debido al fácil acceso ${ }^{7}$.

\section{PUERTO PARALELO}

En la sección anterior se describieron las tres formas más comunes del empleo de la computadora para usarla como un instrumento de registro. Cada una de las cuales tiene sus ventajas y desventajas. Dado que el propósito fundamental de este artículo es el de sugerir el empleo de las computadoras en las instituciones escolares de todos los niveles de educación, el "bus interno" es el menos indicado puesto que se requiere destapar el equipo y hacer conexiones directas en las ranuras disponibles de la tarjeta principal y esto puede generar obstáculos por miedo a producir algún daño en la máquina. Motivo por el cual en este trabajo se estimula el acercamiento al puerto paralelo.

El puerto paralelo se encuentra en la parte posterior del panel de la computadora es fácil diferenciarlo porque usa un conector DB-25 (figura 2) hembra que consta de 25 pínes distribuidos en Entradas y Salidas las cuales se distribuyen de la siguiente forma:

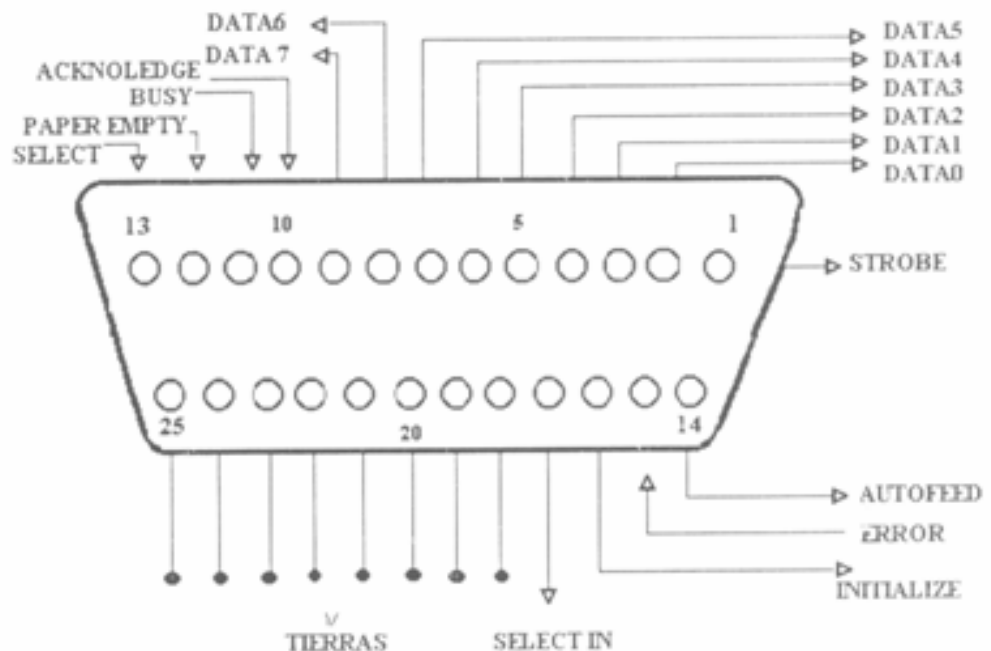

Figura 2. Diagrama esquemático del Conector DB-25 correspondiente al Puerto Paralelo

\footnotetext{
${ }^{6}$ National Semiconductor Corporation. 1995. ADC0808 8-Bit mP A/D Converters with 8-Channel Multiplexer.

${ }^{7}$ CEKIT. 1994. Electrónica \& Computadores. Año 1, N ${ }^{0}$ 3. Pereira, Colombia.
} 
- Las líneas de datos son 8 salidas (van del pin 2 al 9) que se encargan de llevar la información desde la computadora a la impresora, en grupos simultáneos de 8 bits.

- Las Líneas de "status" son cinco entradas (van del pin 10 al 13 y 15 en el conector) y su fin es leer los estados lógicos del conector.

- Las líneas de tierra (van del pin 18 al 25) se ocupan de mantener a un mismo potencial la computadora y el periférico; protegiendo las señales contra ruidos externos.

Es necesario, aclarar que desde la configuración de la computadora a cada puerto paralelo se le han asignado 3 direcciones; ya sea, para entradas o para salidas. Por ejemplo: 3BC (para salidas), 3BD (para entradas) y 3BE (para salidas). Como ya se mencionó; la señal análoga se convierte en una señal de 8 bits que para registrarla, la computadora requeriría de 8 entradas. Si se observa la figura 2, el puerto paralelo sólo cuenta con 5 bits de entrada (líneas 10-13 y 15). Por esta razón, es necesario utilizar un circuito integrado (CD4066), como interruptor, el cual se encarga de dividir la señal en paquetes de 4 bits, que serán registrados uno tras del otro en la memoria de la computadora para así manipularlos según las necesidades (ver figura 3).

Dado el registro de la señal física y una vez convertida en forma digital (ceros y unos); el usuario debe comunicarse con la computadora para poder leer los datos, diseñando un programa que mida los potenciales y que permita llevarlos a la pantalla de la computadora; es decir, se deben adquirir los datos que dan cuenta del fenómeno en si, utilizar un conjunto de instrucciones básicas que operan entre la CPU y la memoria.

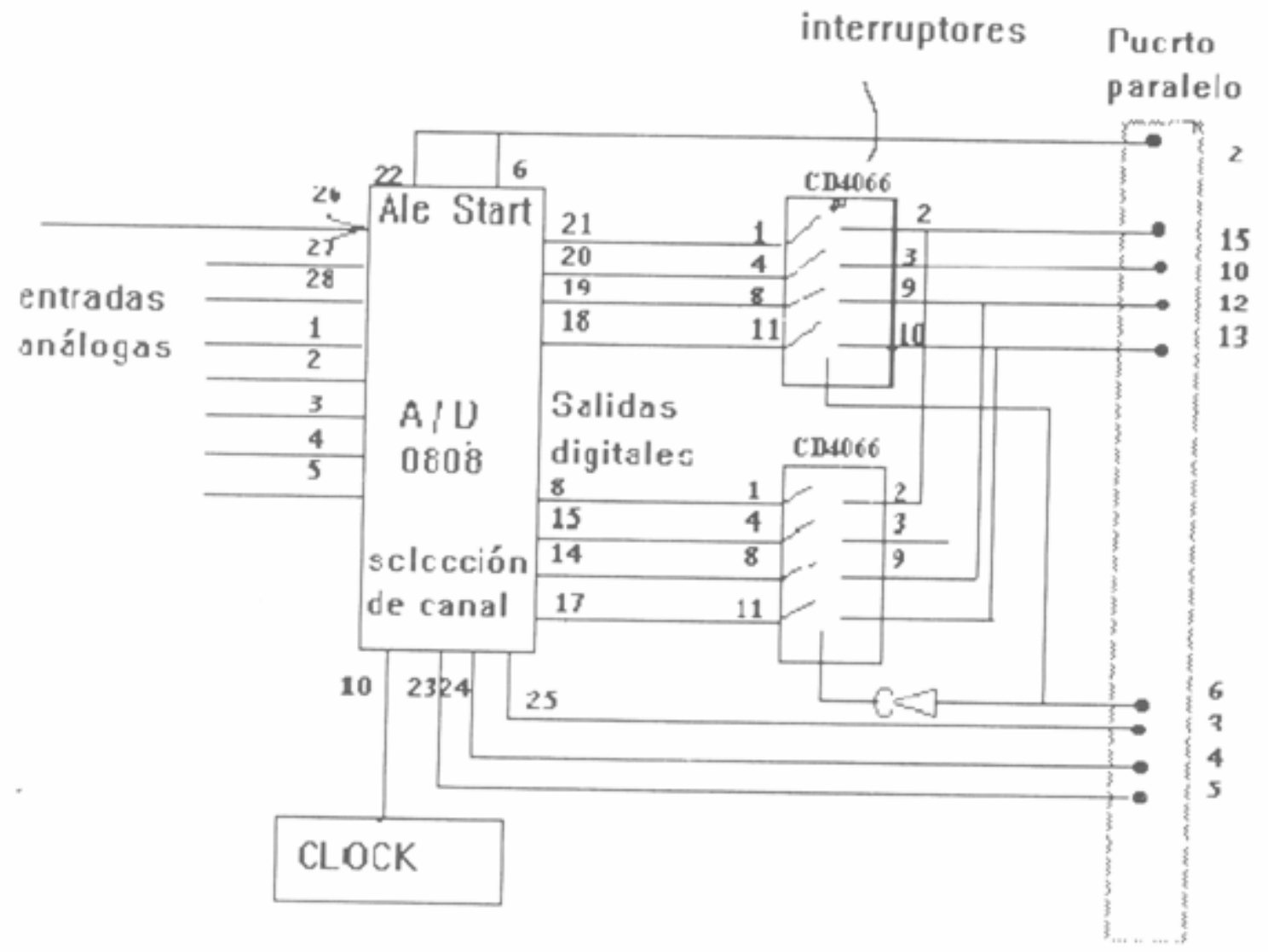

Figura 3. Diagrama esquemático de la entrada de la señal eléctrica al Puerto Paralelo. 


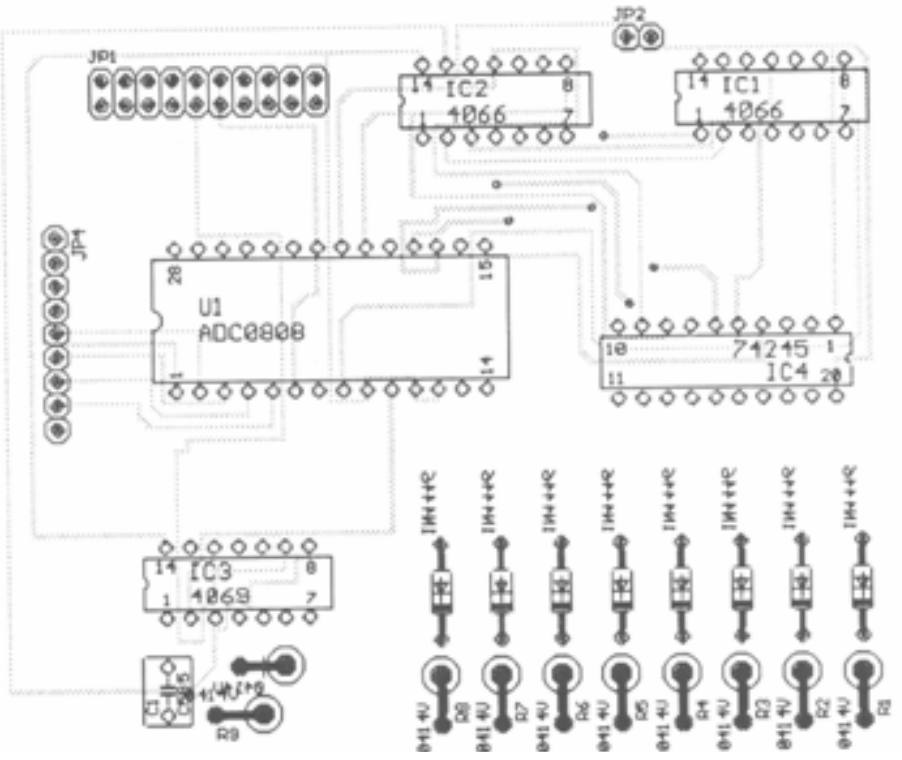

Figura 4. Ubicación de los elementos en la tarjeta. Vista superior

En las figuras 4, 5 y 6 se presentan los circuitos impresos y la localización de los elementos.

En resumen, el montaje completo para lograr el registro y el control de una señal física consta de tres bloques:

- Conversión Análoga-Digital (ADC0808).

- Una vez convertida la señal en una palabra de 8 bits, se fracciona en dos paquetes de cuatro (CD4066) para hacerlos ingresar al puerto por la línea de "Status Port".

- Conexión al puerto paralelo por medio del conector DB-25 macho.

Este montaje puede realizarse sobre un tablero de conexiones (protoboard) o realizar el circuito impreso como el indicado en las figuras 5 y 7.

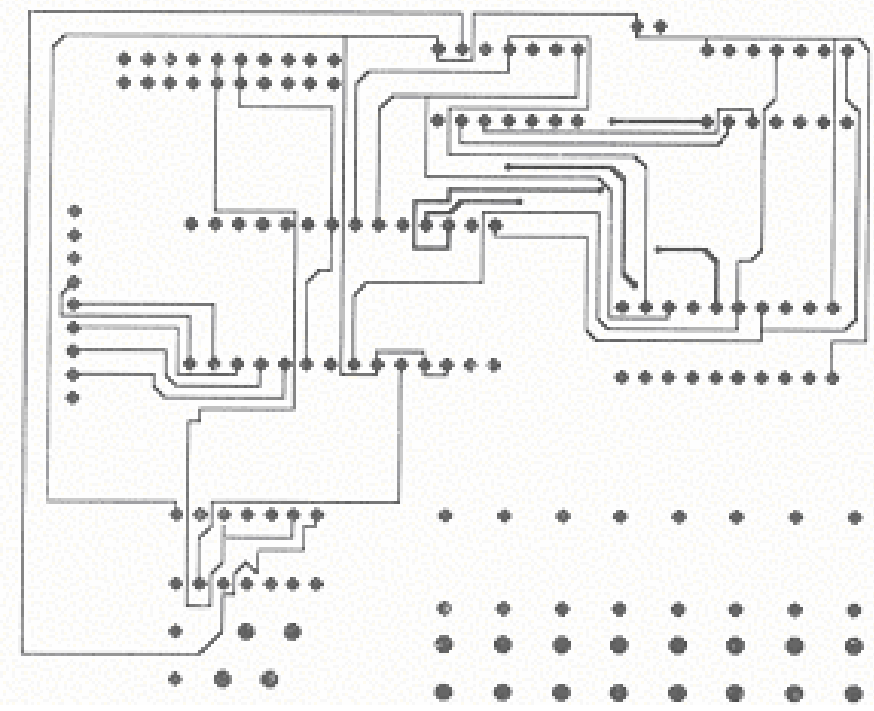

Figura 5. Pistas de la cara superior del circuito impreso. 


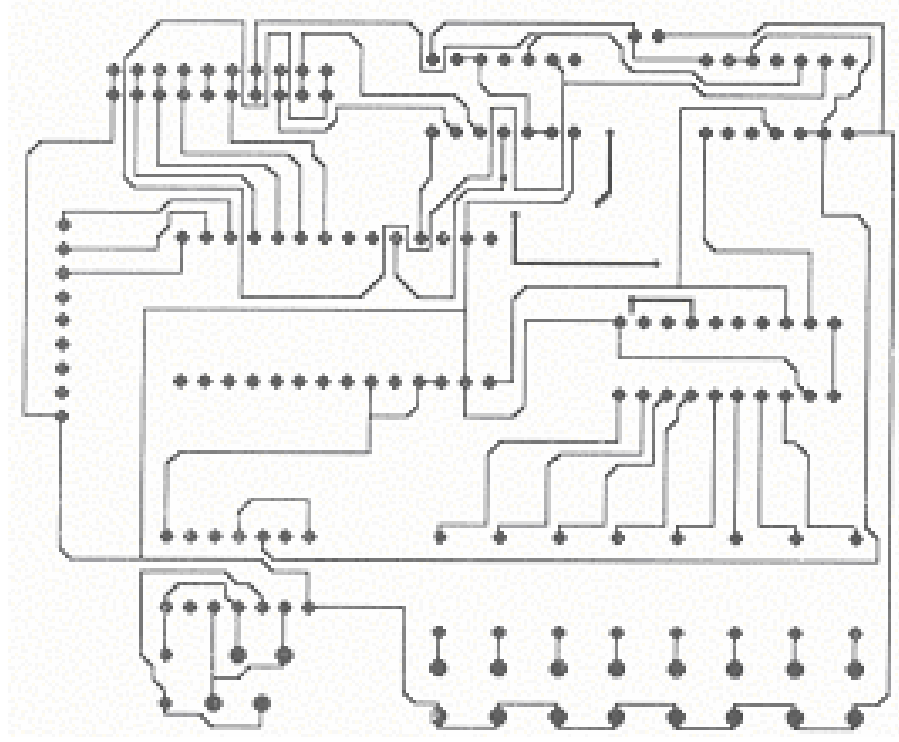

Figura 6. Pistas de la cara inferior del circuito impreso

El siguiente programa (Qbasic) captura datos para las ocho entradas del conversor y los almacena en una matriz de $8 \times 2000$ byts

CLS

DATO. $=\& \mathrm{H} 378$

STATUS DATO +1

CONTROL $=$ DATO +2

CAN 7: CICL 2000

DATOB $=$ O: DATOA $=16$

DIM A(CAN, CICL)

FOR CICLO $=1 \mathrm{TO} \mathrm{CICL}$

FOR CANAL $=0$ TO CAN

FOR T= 0 TO 5: NEXT T

DATOS $=$ CANAL * $2+$ DATOB

OUT DATO, (DATOS + O): REM "fijar el canal a trabajar")

FOR T= TO1: NEXT T

OUT DATO, (DATOS + 1): REM "pulso inicio de conversión"

FOR T = TO1: NEXT T

OUT DATO, (DATOS + O)

FORT 1 TO 1: NEXT T

A INP(STATUS)

A = A AND \&H78: REM "elimina los bits 1,2,3 y 8 de la lectura"

A A / 8: REM "tres rotaciones a derecha"

$\mathrm{A} 1=\mathrm{A}$

DATOS $=0$

DATOS $=$ CANAL ${ }^{*} 2+$ DATOA

OUT DATO, DATOS: REM "valor de la parte alta a leer"

FOR T 0 TO 200

NEXT T

$B=$ INP (STATUS)

$B=B$ AND \&H78: REM "elimina los bits 1,2,3 y 8 de la lectura"

$B=B / 8$

B1 B *16: REM "una rotación a izquierda" 


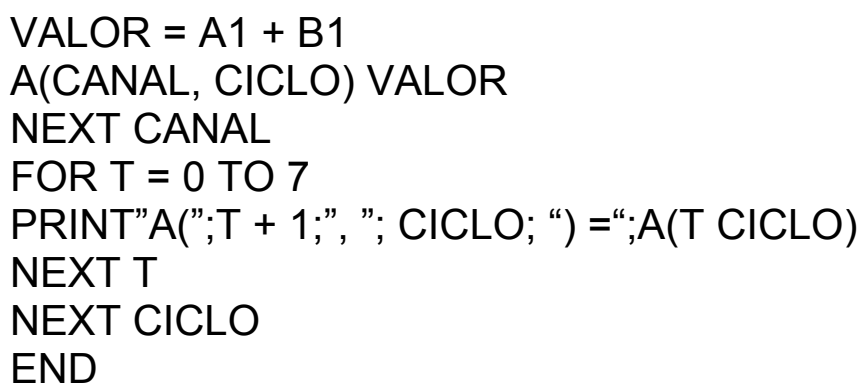

Otro ejercicio interesante (para acercarse al puerto paralelo) consiste en manipular sus 8 salidas; es decir, colocar en as líneas de datos una señal de interés en el campo de la electrónica o del electromagnetismo tales como: una función senoidal, diente de sierra o pulsos cuadrados.

Para registrar un experimento; como por ejemplo la ley de Ohm, es necesario usar tanto las entradas como las salidas del puerto paralelo. Las salidas para enviar órdenes a la interface, por ejemplo iniciar el proceso de conversión de la señal eléctrica en digital y las entradas para que dicha señal digital ingrese a la computadora. Esto se manipula desde el programa a través de las distintas instrucciones del mismo.

\section{CONCLUSIONES}

La computadora, que es básica en el desarrollo de los distintos campos de investigación debe constituirse en apoyo de la teoría y la práctica pedagógica en la institución educativa, ya que, permite a los estudiantes estar en contacto con las realidades físicas que lo rodean en su momento si es usada como instrumento de registro y control de fenómenos; resulta una buena herramienta de apoyo a las prácticas de laboratorio. Pues, aunque el proceso de medida no es tan simple, es asequible a las personas que quieren implementar el puerto paralelo de su computadora realizando procesos de medición y control de variables físicas. De forma que la computadora se convierte en un elemento de ayuda educativa para ser empleado en las instituciones escolares.

Es de advertir que esta propuesta está orientada a darle "un uso más" a las computadoras de las instituciones escolares y particularmente a los equipos XT y AT cuyas salidas del puerto paralelo son unidireccionales. Por esto la necesidad de partir la palabra de entrada en 4 bits, pero en las nuevas computadoras los puertos paralelos vienen con una configuración bidireccional, lo que permite leer la palabra completa de 8 bits. De esta forma se eliminan componentes, conexiones y se hace más rápida la lectura $^{8}$,

Finalmente, con el mejoramiento y producción de nuevos circuitos integrados y particularmente los "Microcontroladores PIC", que además de ofrecer ventajas de economía en espacio y precio, trae incorporado el convertidor análogo-digital y en consecuencia resulta más sencillo construir las interfaces y con relativa facilidad se puede convertir la computadora en un Osciloscopio ${ }^{10}$.

\footnotetext{
${ }^{8}$ CEKIT. 1998. Electrónica \& Computadores. Mayo, $N^{0} 41$. Buenos Aires.

${ }^{9}$ CEK1T. 1998. Electrónica \& Computadores. Julio, N $N^{0}$ 43. Buenos Aires.

${ }^{10}$ CEKIT. 1999. Electrónica \& Computadores. Marzo, № 41. Buenos Aires 